\title{
Atherosclerosis Amelioration by Allicin in Raw Garlic through Gut Microbiota and Trimethylamine- $N$-Oxide Modulation
}

Lee-Yan Sheen ( $\sim$ lysheen@ntu.edu.tw)

National Taiwan University, Taipei, Taiwan

Suraphan Panyod

National Taiwan University, Taipei, Taiwan

Wei-Kai Wu

National Taiwan University Hospital

Pei-Chen Chen

National Taiwan University, Taipei, Taiwan

Kent-Vui Chong

National Taiwan University, Taipei, Taiwan

Yu-Tang Yang

National Taiwan University Hospital

Hsiao-Li Chuang

National Applied Research Laboratories

Chieh-Chang Chen

National Taiwan University Hospital

Rou-An Chen

National Taiwan University

Po-Yu Liu

National Taiwan University https://orcid.org/0000-0003-1290-0850

Ching-Hu Chung

Mackay Medical College

Huai-Syuan Huang

National Taiwan University

Angela Yu-Chen Lin

National Taiwan University

Ting-Chin David Shen

University of Pennsylvania

Kai-Chien Yang

National Taiwan University College of Medicine

Tur-Fu Huang 
National Taiwan University College of Medicine

\section{Cheng-Chih Hsu}

National Taiwan University

Chi-Tang Ho

Rutgers University

\section{Hsien-Li Kao}

National Taiwan University Hospital

\section{Alexander Orekhov}

Skolkovo Innovative Center

\section{Ming-Shiang Wu}

National Taiwan University Hospital

\section{Article}

Keywords: cardiovascular disease, atherosclerosis, garlic juice, allicin, trimethylamine $\mathrm{N}$-oxide, $\mathrm{Y}^{-}$ butyrobetaine, gut microbiota

Posted Date: July 12th, 2021

DOI: https://doi.org/10.21203/rs.3.rs-519786/v1

License: (c) (1) This work is licensed under a Creative Commons Attribution 4.0 International License. Read Full License

Version of Record: A version of this preprint was published at npj Biofilms and Microbiomes on January 27th, 2022. See the published version at https://doi.org/10.1038/s41522-022-00266-3. 


\section{Abstract}

Cardiovascular disease (CVD) is strongly associated with the gut microbiota and its metabolites, including trimethylamine- $N$-oxide (TMAO) formed from $₫$-carnitine. Raw garlic juice, with allicin as its primary compound, has been shown to powerfully impact the gut microbiota. This study validated the benefits of raw garlic juice against CVD risk via modulation of the gut microbiota and its metabolites. Allicin supplementation significantly decreased serum TMAO in $\triangle$-carnitine-fed C57BL/6J mice. It also reduced aortic lesions and altered the fecal microbiota in carnitine-induced, atherosclerosis-prone, apolipoprotein E-deficient mice. In human subjects exhibiting high TMAO production, raw garlic juice intake for a week reduced TMAO formation, improved gut microbial diversity, and increased the relative abundances of beneficial bacteria. In in vitro study, raw garlic juice inhibited $\mathrm{y}$-butyrobetaine (YBB) and trimethylamine (TMA) production by the gut microbiota. Thus, raw garlic juice can potentially prevent cardiovascular disease by decreasing TMAO production through gut microbiota modulation.

\section{Introduction}

Cardiovascular disease (CVD) is a primary cause of global mortality responsible for approximately onethird of all deaths worldwide. ${ }^{1,2}$ Consumption of an unhealthy diet, excessive alcohol use, smoking, and lack of physical activity are considered traditional health risk factors for CVD. ${ }^{3}$ Recently, an insidious CVD risk factor has been explored - the gut microbiota, now considered an endocrine organ that communicates with the human body through the gut-systemic axis and extensively modifiable by foods. 4, 5, 6 The gut microbiota utilizes nutrients from undigested foods for growth and produces miscellaneous metabolites that regulate host-microbe homeostasis, which may be involved in the pathogenesis of

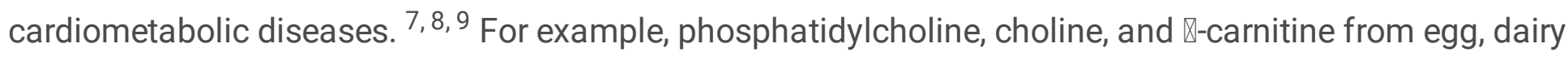
products, and red meat can be metabolized by specific gut bacteria to produce trimethylamine (TMA), which is subsequently oxidized to trimethylamine $\mathrm{N}$-oxide (TMAO) by hepatic flavin monooxygenase. ${ }^{10}$, 11, 12 Increased concentrations of blood TMAO has demonstrated a strong link to increases in major adverse cardiovascular events and all-cause mortality. ${ }^{13,14}$ The mechanisms underlying the atherogenic and thrombogenic effects of TMAO include enhanced foam cell formation, reduced reverse cholesterol transport, and induction of platelet aggregation. ${ }^{9}, 10,12,15$ TMAO has been reported to enhance platelet aggregation and induce thrombosis in both in vitro and human studies. ${ }^{16,17}$ The following multistep microbial pathway was recently proposed as the principal route for TMAO production through carnitine metabolism by the gut microbiota: -carnitine $\rightarrow$-butyrobetaine $(\mathrm{\gamma BB}) \rightarrow$ TMA $\rightarrow$ TMAO. ${ }^{18,19}$ A novel gut bacterium, Emergencia timonensis, was found to be capable of transforming YBB to TMA anaerobically and was reported to partially explain the phenotype of high-TMAO producers among human beings. ${ }^{18,20}$

Investigations on how different food components interact with the gut microbiota have improved our understanding of modifying our dietary behaviors to achieve a health-promoting state. ${ }^{8,21}$ Certain approaches were proposed for reducing TMAO production by the gut microbiota such as antibiotic use, 
fecal microbiota transplantation, and administration of TMA lyase inhibitor. 7, 9, 22, 23, 24 However, these approaches might exhibit safety concerns and require validation in human studies. Garlic (Allium sativum L.) has a long history of use as a spice in human food. ${ }^{25}$ Traditionally, the purpose of adding garlic to meat in processed foods is not only to improve flavor but also to extend shelf life. In herbal medicine, garlic has been used as a dietary therapy against cardiovascular and other metabolic diseases. $26,27,28$, 29 It has been widely used as a natural antibiotic on the basis of its broad-spectrum antimicrobial property. ${ }^{30}$ Allicin is the primary compound in raw garlic puree; it is produced through the conversion of alliin by alliinase when the clove is crushed. ${ }^{31}$ Allicin has been shown to demonstrate various antimicrobial activities in both in vitro and in vivo studies. ${ }^{31,32,33}$ Recently, allicin was reported to exhibit a modulatory effect on the gut microbiota and reduced hepatic steatosis. ${ }^{34,35}$ Additionally, our previous pilot study showed that allicin supplementation shaped the gut microbiota composition and reduced TMAO production by the gut microbiota in mice subjected to moderate carnitine consumption $(0.02 \%$ carnitine). ${ }^{36}$ However, the role of raw garlic with allicin in improving cardiovascular phenotypes via gut microbiota modulation has not been fully elucidated. Therefore, in this study, we aimed to investigate the effect of allicin and raw garlic in modulating both the function and composition of the gut microbiota for cardiovascular protection. We investigated the TMAO-reducing and gut microbiota-modulating effects of allicin in raw garlic in both rodent models and humans with higher carnitine consumption. ${ }^{37} \mathrm{We}$ additionally compared the anti-TMAO and anti-atherosclerotic efficacies of allicin with that of the novel TMA lyase inhibitor 3,3-dimethyl-1-butanol (DMB), ${ }^{24}$ which has been previously shown to reduce serum TMAO in carnitine-treated mice, ${ }^{17}$ through studies in both wild-type and apolipoprotein E-deficient $\left(\mathrm{ApoE}^{-/-}\right)$mice. Finally, we examined the impact of allicin-containing raw garlic juice on the TMAproducing activity of the human gut microbiota and specific gut bacteria responsible for the multistep TMAO production.

\section{Results}

\section{Allicin significantly reduced TMA and TMAO in mice, with less impact on the gut microbiome compared to \-carnitine.}

To investigate the protective effect of allicin against CVD, we subjected C57BL/6J mice to increased daily

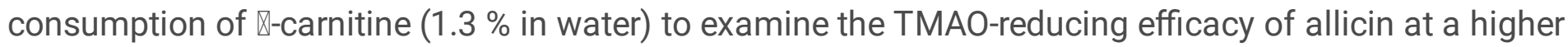
carnitine dosage. Additionally, we compared the TMAO-reducing effect of allicin with DMB (Fig. 1a). There was no significant change in the bodyweight of the mice (Supplementary Fig. 1). Serum TMA and TMAO were significantly reduced in the allicin-supplemented groups than in the carnitine treatment group $(P=0.011$ and $P=0.0008$, respectively) (Fig. $1 b-c)$. Additionally, serum $\gamma B B$ was reduced in the allicinsupplemented group (Fig. 1d). According to the microbiome analysis, shift in the overall microbial

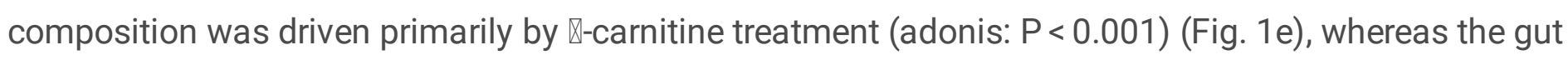
microbiota composition did not vary considerably among the carnitine, carnitine + allicin, and carnitine + DMB groups. Additionally, the observed operational taxonomic units (OTUs) and Chao1 index were 
decreased in the $₫$-carnitine treatment groups $(P=0.0034 ; P=0.0401$, respectively), whereas supplementation with allicin and DMB did not reverse the alpha diversity (Fig. $1 \mathrm{f}$ and Supplementary Fig. 2b). The family-level relative abundance bar plot and OTU-level heatmap supported that $₫$-carnitine primarily shaped the gut microbiota composition with respect to specific taxa (Fig. $1 \mathrm{~g}$ and Supplementary Fig. 2c).

\section{Raw garlic juice reduced TMAO-producing capacity and modified the gut microbiome by enriching beneficial gut bacteria in humans.}

On the basis of the TMAO-reducing effects of allicin in previous murine studies, we conducted a human pilot study to investigate whether raw garlic juice at an allicin-equivalent dosage could reduce the TMAOproducing capacity in the human body. We utilized an oral carnitine challenge test (OCCT) to evaluate TMAO-producing capacity in humans. ${ }^{37}$ Nine volunteers were recruited to receive the OCCT, and those defined as high-TMAO producers (plasma TMAO $\mathrm{MAX} \geq 10 \mu \mathrm{M}$ ) according to the OCCT underwent the garlic juice intervention. Among the nine volunteers, seven participants were determined to be high-TMAO producers (Plasma TMAO $\mathrm{MAX}=130.91,82.28,57.53,22.84,135.62,44.41$, and $21.31 \mu \mathrm{M}$ ) (Fig. 2a and Supplementary Fig. 3a) and were allowed to proceed to the garlic juice intervention study. We first converted the allicin dose used in the murine study into a dose for humans and calculated the amount of blended garlic juice containing the equivalent dose of allicin for the interventional study. The blended raw garlic juice used in this pilot study contained $0.89 \mathrm{mg} / \mathrm{mL}$ allicin, and the participants consumed $55 \mathrm{~mL}$ of the prepared garlic juice per day for a week (Supplementary Fig. 4). All participants received a second OCCT after the one-week intervention. For the comparison of TMAO-producing capacity before and after the garlic juice intervention, a trend of reduced plasma $T M A O_{M A X}$ and $T M A O_{A U C}$ was observed $(P=0.051$; $P=0.081$ ) (Fig. 2b). Notably, both urine $T_{M A O}$ MAX and $T_{M A O}$ AUC were significantly reduced after the oneweek garlic juice intervention $(P=0.0252 ; P=0.0248)$ (Fig. 2c). The strong correlation between plasma and urine TMAO concentrations (Supplementary Fig. 5a) supports the use of urine samples for measuring TMAO-producing capacity. Interestingly, plasma levels of $\mathrm{YBB}$ in the OCCT were significantly increased $(P<0.01)$ (Fig. 2d), suggesting that the bioactive compounds in garlic may prevent the microbial transformation of $ү B B$ into TMA, thus decreasing TMAO formation. Our study showed a substantial reduction in platelet aggregation following the garlic juice intervention. Additionally, the maximum amplitude of platelet aggregation tended to exhibit a positive correlation with the TMAO levels (Supplementary Fig. 5b-c). Intervention with garlic juice did not lead to toxicity based on plasma biochemistry results (Supplementary Table 1). Only aspartate aminotransferase (AST) and alanine aminotransferase (ALT) were slightly increased but remained within normal range.

Overall, the gut microbial composition was not altered by the one-week consumption of garlic juice, as demonstrated through principal coordinate analysis (PCOA). The intra-individual dissimilarity in the gut microbiome before and after treatment was considerably lower than the inter-individual index $(P<0.01)$, suggesting that a relatively minor portion of the gut microbiota was altered by the garlic juice intervention (Fig. 2e and Supplementary Fig. 6a). Regarding the alpha diversity, garlic juice intervention significantly 
increased the Shannon index $(P=0.0304)$ (Fig. 2f). At the family level, the fecal microbiota was altered by garlic juice; the constituent families included Acidaminococcaceae, Akkermansiaceae, Burkholderiaceae, and Erysipelotrichaceae (Fig. 2g). At the genus level, after the garlic juice intervention, the fecal microbiota of high-TMAO producers was comparatively enriched in Akkermansia, Desulfovibrio, Lachnospiraceae UCG - 008, UBA1819, and Christensenellaceae $R-7$ group (Supplementary Fig. 7). The volcano plot showed that 13 taxa were significantly altered by the garlic-juice intervention (Fig. $2 \mathrm{~h}$ ). Interestingly, certain beneficial and anti-inflammatory gut commensal bacteria, including Faecalibacterium prausnitzii and Akkermansia spp., were significantly enriched after the one-week garlicjuice intervention ( $q<0.05)$ (Fig. 2i).

\section{Allicin ameliorated atherosclerosis, reduced TMATMAO production, and partially reversed the microbiome shifts in carnitine-treated ApoE ${ }^{-/-}$mice.}

Our human study demonstrated that raw garlic juice potentially reduced TMAO production and regulated the gut microbiome by enriching beneficial gut bacteria. Thus, we further evaluated the plaque-inhibiting effects of allicin and compared it with $\mathrm{DMB}$ in the carnitine-induced atherosclerotic $\mathrm{ApoE}^{-/-}$murine

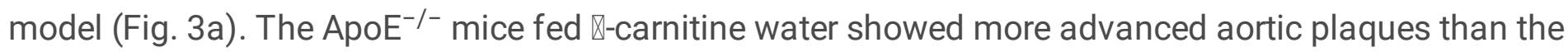
control group $(p<0.0001)$. Both allicin and DMB significantly reduced aortic plaques $(P=0.008$ and $p<$ 0.0001) (Fig. 3b-c). We additionally performed an isotope-labeled $d_{9}$-carnitine challenge test to specifically evaluate the effects of allicin on $\mathrm{d}_{9}-T M A / d_{9}-T M A O$ producing ability from the gut microbiota. The levels of both $d_{9}$-TMA and $d_{9}$-TMAO in the carnitine group were considerably elevated, and their area under the curve (AUC) values were significantly higher compared with that in the control group $(P=$ 0.0006 and $P=0.0008$, respectively). Both serum $d_{9}-$ TMA and $d_{9}$-TMAO were substantially reduced in mice of both carnitine + allicin and carnitine + DMB groups compared with mice fed carnitine alone. Additionally, the control + allicin group showed lower $d_{9}$-TMA and $d_{9}$-TMAO levels than the control group (Fig. 3d-e). Although the carnitine + allicin treatment in the $\mathrm{ApoE}^{-/-}$mice showed significantly reduced atherosclerotic plaques compared with carnitine treatment alone, the reduction in TMAO-producing capacity and serum TMAO did not exhibit the expected significant difference. As this is the first time that allicin was administered to $\mathrm{ApoE}^{-/-}$mice over a 16-week period, we speculate that the mouse strain and treatment duration of allicin may influence the effects of allicin on TMAO reduction. Additionally, $d_{9}-\gamma B B$, an intermediate of $d_{9}$-carnitine metabolism by gut microbiota, increased significantly in the carnitine group compared with that in the control group $(P=0.047)$, which is consistent with the result from our previous human study (Fig. $3 f$ and Fig. 2c). Interestingly, the carnitine + allicin and carnitine + DMB groups showed greater elevation in serum $d_{9}-\gamma B B$ than the carnitine group, which indicates that the process of $d_{9}-$ үBB transformation into downstream TMA might be inhibited, leading to the buildup of d9-үBB.

We subsequently investigated the alteration in gut microbiome composition in different treatment groups through 16S rRNA sequencing analysis with the QIIME pipeline. Regarding the beta-diversity, the PCoA showed that carnitine water induced a significant microbiome shift (especially at the PCoA2 axis) that

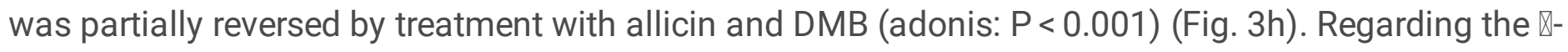


diversity, carnitine water alone exhibited decreased observed OTUs, the Shannon index, and the Chao1 index $(P=0.0004 ; P<0.001 ; P=0.0063$ respectively), whereas additional treatment with allicin or $D M B$ showed favorable alpha-diversity indices (Fig. $3 \mathrm{~g}$ ). A heatmap of the gut microbiome demonstrated differences in the fecal microbiome (Fig. 3i); the carnitine + DMB group exhibited enrichment in certain beneficial bacteria, including Roseburia and Akkermansia, which have been reported to correlate inversely

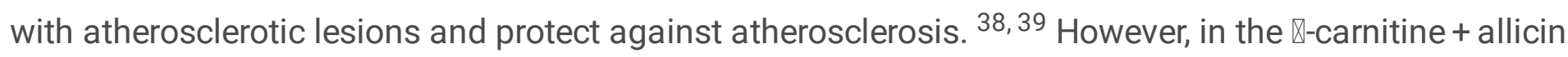

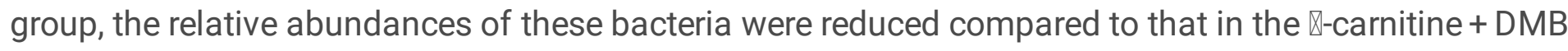
group, suggesting that allicin might exert bactericidal activity against these beneficial bacteria and limit

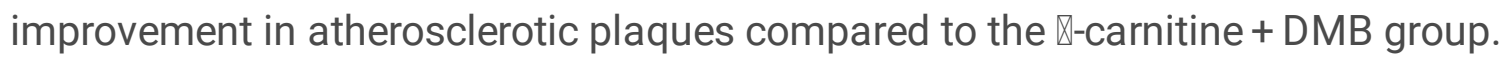

\section{Raw garlic juice inhibited microbial carnitine $\rightarrow$ YBB $\rightarrow$ TMA pathways in vitro}

Our study showed that raw garlic juice reduced TMAO-producing capacity through carnitine metabolism in TMAO-producing participants, C57BL/6J mice, and aortic lesion-exhibiting $\mathrm{ApoE}^{-/-}$mice. However, it is unknown whether it can specifically inhibit microbial pathways of carnitine metabolism. Thus, we subsequently conducted an in vitro study to examine the inhibitory effects of raw garlic juice on the following pathway: carnitine $\rightarrow$ yBB $\rightarrow$ TMA (Fig. 4a). In functional assays, we used fecal samples from the high-TMAO producers and documented strain types responsible for the following pathways: carnitine $\rightarrow$ үBB and $\mathrm{\gamma BB} \rightarrow \mathrm{TMA}$.

We standardized the functional assay to examine inhibition of the following pathway: carnitine $\rightarrow \gamma B B \rightarrow$ TMA. For this purpose, we established a simplified model by co-culturing type strains that have been reported to metabolize carnitine to form $\mathrm{YBB}$, including Proteus penneri, Escherichia fergusonii, and Edwardsiella tarda. The three co-cultured bacterial strains were inoculated in Wilkins-Chalgren (WC) broth supplemented with $d_{9}$-carnitine. The concentration of garlic juice was titrated to a potent inhibitory level in the incubation broth. Raw garlic juice at $5 \%$ and $10 \%$ exhibited potent inhibition of the transformation of $d_{9}$-carnitine into $d_{9}-\gamma B B$ (Fig. $4 b$ ). Single type strain experiments showed consistent results (Supplementary Fig. 10). E. timonensis is a novel bacterium isolated from the human gut; it was recently reported to metabolize $\mathrm{YBB}$ to TMA. We observed that raw garlic juice exhibited potent inhibition of $d_{9}$-TMA production by $E$. timonensis using $d_{9}-\gamma B B$ as a substrate at $0.6 \%$ garlic juice, which is a relatively low concentration of the prepared garlic juice (Fig. 4c).

To test the inhibitory effect of garlic juice in a complex microbiome community, we collected the feces from high-TMAO producing participants and cultured in WC broth supplemented with $d_{9}$-carnitine or $d_{9}$ YBB. WC broth supplemented with $d_{9}$-carnitine inoculated with feces from high-TMAO producers showed that the $d_{9}-\gamma B B$ level was the highest at $6 \mathrm{~h}$ while $d_{9}-T M A$ was not detected at that time point. At $12 \mathrm{~h}$, the $d_{9}-\gamma B B$ level was reduced with simultaneous formation of $d_{9}-T M A$. Raw garlic juice at $5 \%$ and $10 \%$ concentrations in the culture medium showed optimal inhibition of TMA formation with moderate bacterial growth inhibition (Fig. 4d). $d_{9}-\gamma B B$ started to be utilized to form $d_{9}$-TMA over 6 to $12 \mathrm{~h}$ in broth 
supplemented with $d_{9}-\gamma B B$. Similarly, $5 \%$ and $10 \%$ garlic juice were determined to be an optimal concentration for preventing fecal microbiota from utilizing $d_{9}-\gamma B B$ to form $d_{9}$-TMA (Fig. 4e).

\section{Discussion}

Allicin supplementation in C57BL/6J mice fed a high concentration (1.3\%) of 『-carnitine demonstrated that the primary compound in raw garlic juice, allicin, potentially inhibited the gut microbiota- and liverderived TMA and TMAO in circulation, which is consistent with the results of our previous study, in which

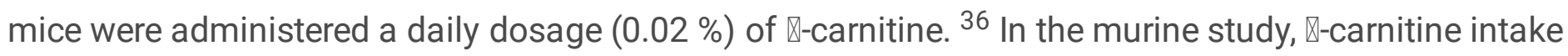
was the principal factor affecting the overall gut microbiota composition compared with allicin or DMB intervention. The TMAO-reducing effect of allicin is probably based on its broad-spectrum antimicrobial activities. ${ }^{31}$ Additionally, previous studies reported that garlic and its bioactive compounds exhibited lipid-lowering and fatty liver-protective effects via gut microbiota modulation. ${ }^{35,40}$ Following allicin intake, it can be metabolized to various sulfur-containing bioactive compounds, such as diallyl sulfide, diallyl disulfide, and diallyl trisulfide for modulating the microbiome composition in the gut. $41,42,43,44$

Previously, in a human study, we developed the OCCT, which can robustly distinguish the low- and highTMAO producers. ${ }^{37}$ In this study, high-TMAO producers were defined as individuals exhibiting OCCT plasma $\mathrm{TMAO}_{\mathrm{MAX}}$ level $\geq 10 \mu \mathrm{M}^{20}$. There is currently a lack of studies on the effect of garlic on the gut microbiota in the human body; this study is the first to demonstrate the impact of garlic on TMAO production by the gut microbiota. In this human study, after the intake of garlic juice for one week, both plasma and urine TMAO were reduced, suggesting that the bioactive compounds in garlic inhibited gut microbial carnitine utilization and TMA-formation ability, resulting in reduction of TMAO. The current study demonstrated that plasma and urine TMAO exhibited a high positive correlation that was consistent with the results from previous studies. ${ }^{20,37}$ Currently, the primary genes responsible for converting carnitine to TMA by the gut microbiota remains unclear. A Rieske-type microbial $C n t A / B$ enzyme was reported to convert carnitine to TMA. ${ }^{15}$ However, it is doubtful whether $C n t A / B$ is responsible for TMA formation in the gut anaerobic environment because oxygen is required for its

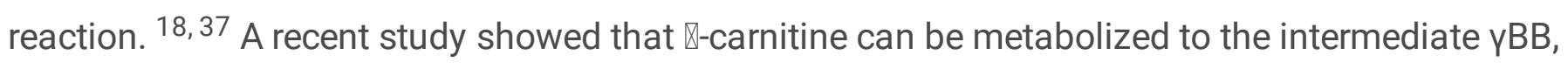
followed by transformation into TMA via a low-abundance anaerobic bacterium called $E$. timonensis; this could be the major pathway for TMA generation by the gut microbiota in the oxygen-limited environment. 18 This study showed an increase in plasma $ү B B$ following the garlic-juice intervention, suggesting that garlic juice might block the conversion of $\gamma B B$ to TMA, resulting in accumulation of $\gamma B B$.

The gut microbiome analysis showed that the garlic-juice intervention increased the Shannon index, indicating that a more diverse and balanced microbial community was produced by garlic-juice consumption. The overall gut microbiome profile for each individual was moderately affected by the garlic-juice intervention; however, the relative abundances of certain beneficial gut bacteria were increased following garlic-juice intake, including Akkermansia, Lachnospiraceae, Christensenellaceae, and Faecalibacterium prausnitzii. Akkermansia muciniphila, a mucin-degrading bacterium that resides in 
the mucus layer, has been demonstrated to be inversely associated with obesity and type 2 diabetes. Prebiotic administration with Akkermansia improves the metabolic profile and reduces metabolic disorders, obesity, endotoxemia, adipose tissue inflammation, and insulin resistance. ${ }^{45} \mathrm{~A}$. muciniphiladerived extracellular vesicles improve gut permeability through the regulation of tight junctions, as well as decrease body weight gain and reduce glucose tolerance in high-fat diet-induced diabetic mice. ${ }^{46}$ Furthermore, it shields against atherosclerosis by blocking metabolic endotoxemia-induced inflammation in $\mathrm{ApoE}^{-/-}$mice. ${ }^{39}$ The garlic intervention increased the level of Faecalibacterium pranusnitzii, which has been reported to be an anti-inflammatory commensal bacterium that secretes a metabolite blocking NF$\mathrm{KB}$ activation and IL-8 secretion. ${ }^{47}$ These results suggested that the garlic-juice intervention modulates the gut microbiota of high-TMAO producers in a favorable direction. However, drinking raw garlic juice on an empty stomach may cause abdominal discomfort, and thus it should be consumed with a meal to prevent undesirable stomachache.

In $\mathrm{ApoE}^{-/-}$mice, $1.3 \%$ «-carnitine induced atherosclerosis that was ameliorated by allicin supplementation, indicating the potential anti-atherosclerotic activity of allicin. However, we observed that TMAO inhibition by allicin was more effective with short-term treatment in C57BL/6J mice than with longterm treatment in $\mathrm{ApoE}^{-/-}$mice, which suggested that long-term allicin administration could result in the resistance of TMA-producing bacteria against the anti-microbial effect of allicin. However, long-term allicin administration resulted in more differences in the overall gut microbiota in $\mathrm{ApoE}^{-/-}$mice. The allicin and DMB group with $₫$-carnitine group in the long-term study exhibited a similar gut microbiota composition. Additionally, the in vitro study suggested that garlic inhibited the formation of both $d_{9}-\gamma B B$ and $\mathrm{d}_{9}$-TMA in the culture medium inoculated with the co-culture of bacteria producing YBB (P. penneri, $E$. fergusonii, and E. tarda) and TMA (E. timonensis), as well as inhibited TMA formation by the complex bacterial consortium of the TMAO-producing microbiota.

Here, we demonstrated that supplementation with allicin inhibited TMAO production through carnitine metabolism by the gut microbiota in both mice and humans and prevented carnitine-induced atherosclerosis in $\mathrm{ApoE}^{-/-}$mice. This was supported by in vitro assays using specific bacteria that metabolize $₫$-carnitine to $\mathrm{YBB}$ and $\mathrm{YBB}$ to TMA (Fig. 5). These data provide valuable evidence that raw garlic, which contains allicin, shifts the gut microbiota composition and modulates the following gut microbial pathway: $₫$-carnitine $\rightarrow$ YBB $\rightarrow$ TMA. In summary, this study suggests that garlic might serve as a potential dietary intervention for CVD prevention.

\section{Methods}

\section{Allicin preparation for the animal study.}

Allicin synthesis, purification, and identification were based on our laboratory protocol. ${ }^{35,36,48}$ The purity of allicin was more than $97 \%$; it was dissolved in $0.5 \%$ carboxymethyl cellulose (CMC) and stored at -80 ${ }^{\circ} \mathrm{C}$ before administration to the mice. 


\section{[-carnitine-fed C57BL/6J mouse model.}

The handling of animals complied with the guidelines of the Institutional Animal Care and Use Committee of National Taiwan University (approval number: NTU107-EL-00170 and NTU107-EL-00084). Six-week-old male C57BL/6J mice were purchased from Taiwan National Laboratory Animal Center. The mice were housed in a room with a $12 \mathrm{~h} \mathrm{light-dark}$ cycle at $22 \pm 2{ }^{\circ} \mathrm{C}$ after an adaptation period of 2 weeks. The mice were randomly divided into experimental groups (i) control; (ii) control + allicin; (iii) carnitine; (iv) carnitine + allicin; and (v) carnitine + DMB. For the carnitine intake group, $1.3 \%$-carnitine in water was supplied. ${ }^{10}$ Allicin treatment groups were orally administered $10 \mathrm{mg} /(\mathrm{kg}$ bw day) allicin in 0.5 $\%$ CMC. ${ }^{36}$ The DMB group was treated with $1 \%$ DMB in water. ${ }^{24}$ The mice were sacrificed by $\mathrm{CO}_{2}$ asphyxiation after two weeks. Blood was collected from the major arteries of the abdomen using a syringe.

\section{Long-term 0 -carnitine-induced atherosclerosis $\mathrm{ApoE}^{-/-}$mouse model.}

The $\mathrm{ApoE}^{-/-}$mice were initially purchased from the Jackson Laboratory (Bar Harbor, ME) and bred in our laboratory animal room according to the guidelines of the Institutional Animal Care and Use Committee of National Taiwan University. Six-week-old female $\mathrm{ApoE}^{-/-}$mice were utilized for the experiment after an adaptation period of 2 weeks. The mice were grouped and treated similarly to the short-term C57BL/6J mouse model, as described previously. The $\mathrm{ApoE}^{-/-}$mice were sacrificed after 15 weeks. Blood was collected from the major arteries of the abdomen using a syringe. Phosphate-buffered saline (PBS) was used to gently flush the aorta to remove blood clots, and the aorta was removed from the body.

\section{Oral $d_{9}$-carnitine challenge test in the mouse.}

On the day of the sacrifice, the mice were gavaged with $200 \mu \mathrm{L}(150 \mathrm{mM})$ of $d_{9}$-carnitine, followed by serial blood collection at $0,4,8$, and 10 h. ${ }^{36}$ Subsequently, the mice were subjected to carbon dioxide asphyxiation. Blood was collected from the major arteries of the abdomen using a syringe at $12 \mathrm{~h}$. The blood samples were centrifuged at $1,000 \times g$ for $15 \mathrm{~min}$. The supernatants were collected and stored at $-80^{\circ} \mathrm{C}$ before further analysis.

\section{Oil red staining of the aorta}

Aortic specimens were rinsed with PBS to remove excess blood residue. Dissection was performed under the microscope using micro-dissection scissors and forceps to remove the adipose tissue. The aorta was fixed with $10 \%$ formalin and stained with oil red 0 dye ${ }^{49}$ as follows: it was (1) washed with deionized water for $5 \mathrm{~min}$; (2) soaked in propylene glycol for $10 \mathrm{~min}$; (3) stained with oil red 0 dye; (4) soaked in 85 $\%$ propylene glycol for $3 \mathrm{~min}$; and (5) washed twice with deionized water. We captured images of the stained aorta and analyzed the aortic lesion area using the Image $\mathrm{J}$ software (Version 1.8.0).

\section{Mouse blood biochemistry analysis}


The serum was extracted via centrifuging the blood at $1,000 \times g$ for 15 min at $4{ }^{\circ} \mathrm{C}$. We analyzed serum biochemical parameters, including total cholesterol, total triglyceride, high-density lipoprotein (HDL-c), AST, and ALT, using commercial test strips (Spotchem II reagent strips; Arkray Inc., Kyoto, Japan) in an automatic blood analyzer (Spotchem EZ). 50

\section{High-TMAO producer screening by OCCT.}

This research was approved by the Research Ethics Committee of National Taiwan University Hospital (201712031RIND), and the study has been registered on ClinicalTrials.gov as NCT04545879. Healthy participants were recruited ( $n=9)$, under the following criteria: (1) age $\geq 20$ years old; (2) no exposure to antibiotics, probiotics, or carnitine supplements within the previous month; (3) no history of chronic diseases including, diabetes mellitus, myasthenia gravis, chronic renal disease, hyperparathyroidism, epilepsy, and severe anemia; (4) Participants were excluded from the study if they reported recent gastrointestinal discomfort (such as abdominal pain or diarrhea).

We used the OCCT, which was previously shown to exhibit better efficacy than fasting plasma TMAO, to identify the TMAO producer phenotype. ${ }^{37}$ All participants fasted at least $8 \mathrm{~h}$ before the OCCT. The

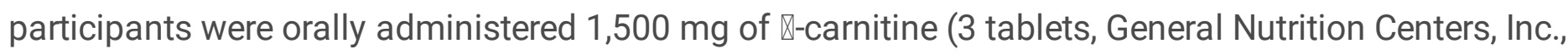
USA). The blood and urine of the participants were collected at $0,24,48$, and $72 \mathrm{~h}$ after carnitine intake. The plasma and urine samples were centrifuged at $1,000 \times g$ for $15 \mathrm{~min}$ and stored at $-80{ }^{\circ} \mathrm{C}$ before the following analyses. Participants with plasma TMAO $\geq 10 \mu \mathrm{M}$ after the OCCT were defined as high-TMAO producers and allowed to proceed for the garlic juice intervention. ${ }^{20}$

\section{Garlic juice preparation and allicin content quantification}

Raw garlic was peeled to remove the skin; $100 \mathrm{~g}$ of garlic and $300 \mathrm{~mL}$ of water (ratio 1:3) were mixed and blended into garlic juice using a blender. After filtration, the raw garlic juice was placed into a glass bottle (55 $\mathrm{mL}$ for each), ready to be consumed by the subjects. The garlic juice required was prepared at once and frozen at $-20^{\circ} \mathrm{C}$ before use.

The allicin content in the raw garlic juice was determined using HPLC (JASCO LC-Netll/ADC/JASCO UV2075 Plus) with Shiseido C18 (5 $\mathrm{m}, 4.6 \mathrm{~mm} \times 250 \mathrm{~mm})$. The mobile phase was deionized water and methanol, and the flow rate was $1 \mathrm{~mL} / \mathrm{min}$. The gradient program was run as follows: $0-100 \%$ methanol, 0-30 min and $100 \%$ methanol, $10 \mathrm{~min}$. The absorbance was detected at $254 \mathrm{~nm}$. The allicin concentration in raw garlic juice was calculated against the allicin standard curve at concentrations of $0.2,0.4,0.6,0.8$, and $1.0 \mathrm{mg} / \mathrm{mL}$.

\section{Garlic Juice Intervention}

High-TMAO producers $(\mathrm{n}=7)$ were asked to consume $55 \mathrm{~mL}$ of raw garlic juice (48 mg of allicin equivalent) once a day during dinner for one week. High-TMAO producers were suggested to consume the garlic juice with a meal to decrease stomach irritation caused by quick drinking of raw garlic juice. The 
high-TMAO producers were free to choose their diet with no restriction on the type of food. After one week of raw garlic juice intervention, the second OCCT was performed.

\section{Gut microbiota inoculum preparation from human feces for in vitro study}

We mixed $1 \mathrm{~g}$ of raw fecal sample with $10 \mathrm{~mL}$ of PBS supplement with $0.05 \%$-cysteine in a tube containing glass beads, homogenized the mixture by vortexing for $1 \mathrm{~min}$ in an anaerobic chamber (Whitley DG250 Workstation, Don Whitley Scientific Limited, UK), filtered the homogenized fecal liquid through a Falcon ${ }^{\circledR} 100 \mu \mathrm{m}$ cell strainer, and subsequently used the fecal filtrate for the study.

\section{Media selection study for maximal TMA production}

To investigate the suitability of the culturing media for producing maximal TMA concentration, we compared three media: gut microbiota medium (GMM), ${ }^{51}$ MEGA, ${ }^{52}$ and WC medium. ${ }^{53}$ Furthermore, we modified these media as a carbon-reduced medium. The media were supplemented with $100 \mu \mathrm{M} \mathbb{\mathrm { B }}$ carnitine. The media recipes are provided in the Supplementary Tables 2-4. Two gut microbiota inocula from the feces of high-TMAO producers were prepared by mixing $1 \mathrm{~g}$ of feces with $15 \mathrm{~mL}$ of PBS with $0.05 \%$ cysteine and filtering the mixture through a $100 \mu \mathrm{m}$ Falcon cell strainer, following which $100 \mu \mathrm{L}$ of the stool filtrate was transferred to $1.9 \mathrm{~mL}$ of medium and subsequently incubated at $37^{\circ} \mathrm{C}$ under anaerobic conditions $\left(80 \% \mathrm{~N}_{2}, 10 \% \mathrm{CO}_{2}\right.$, and $\left.10 \% \mathrm{H}_{2}\right)$ for $0,6,12$, and $24 \mathrm{~h}$. The cultures were collected, their growth measured at optical density (OD) $600 \mathrm{~nm}$ (Spectronic Helios Gamma UV-Vis Spectrophotometer, Thermo Fisher Scientific, UK), centrifuged at 6,000 rpm for $1 \mathrm{~min}$, and the supernatant collected for measuring TMA, TMAO, үBB, carnitine, and choline concentration using LC-MS.

\section{Gut microbiota inoculum and bacteria}

The medium used in this study was WC broth supplemented with $d_{9}$-carnitine or $d_{9}$-үBB. We used the WC broth because it produces the highest TMA level compared with GMM, the MEGA medium, and its carbonreduced medium (Supplementary Fig. 9). The high-TMAO producer stool for the inhibition study was recollected from the participant (GJ07), which exhibited the highest TMAO MAX during the OCCT and possessed the $E$. timonensis $16 \mathrm{~S}$ sequence in the sample. The bacteria used in this study include the bacteria responsible for converting \-carnitine to YBB P. penneri ATCC33519, E. fergusonii ATCC35469, and E. tarda ATCC15947. ${ }^{54}$ E. timonensis DSM101844 transforms $\gamma$ BB to TMA. ${ }^{18}$ A single colony on the WC agar plate of the bacteria was inoculated into the WC broth for $24 \mathrm{~h}$ under anaerobic conditions before being used in the inhibition study.

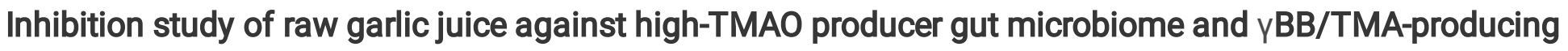 bacteria}

Garlic juice was prepared as described above, following which it was sterilized by filtration through 0.22 $\mu \mathrm{m}$ polyvinylidene fluoride membranes. We inoculated $50 \mu \mathrm{L}$ of the gut microbiota inoculum from the high-TMAO producers, which was prepared as described above, in $1 \mathrm{~mL}$ of WC broth with $50 \mu \mathrm{M} \mathrm{d} 9^{-}$ 
carnitine or $d_{9}-\gamma B B$. Subsequently, the sterilized raw garlic juice was added at final concentrations of 0 , $0.3,0.6,1.3,2.5,5.0$, and $10.0 \%$ and incubated at $37^{\circ} \mathrm{C}$ under anaerobic conditions $\left(80 \% \mathrm{~N}_{2}, 10 \% \mathrm{CO}_{2}\right.$, and $10 \% \mathrm{H}_{2}$ ) for $0,6,12$, and $24 \mathrm{~h}$. We collected the cultures, estimated the growth $\mathrm{OD}_{600}$, centrifuged the cultures at 6,000 rpm for $1 \mathrm{~min}$, and collected the supernatant for measuring $d_{9}-T M A, d_{9}-\gamma B B$, and $d_{9}$ carnitine using LC-MS. For evaluating the inhibition of bacteria related to CVD, the YBB-producing bacteria P. penneri, E. fergusonii, and E. tarda were mixed before the inhibition analysis. We inoculated $100 \mu \mathrm{L}$ of the yBB-producing bacterial mixture in $1 \mathrm{~mL}$ of $\mathrm{WC}$ broth with $100 \mu \mathrm{M} \mathrm{d}$-carnitine, added garlic juice, cultured the bacteria, and performed the analysis as mentioned above. For analyzing the inhibition of $E$. timonensis, we inoculated $100 \mu \mathrm{L}$ of the inoculum in $1 \mathrm{~mL}$ of WC broth with $100 \mu \mathrm{M} \mathrm{d}_{9}-\gamma B B$, added the garlic juice, cultured the bacteria, and performed the analysis as mentioned above.

\section{Measurement of the concentration of TMA, $d_{9}-T M A, T M A O, d_{9}-T M A O, g B B, d_{9}-g B B$, carnitine, $d_{9}$-carnitine, and choline by LC-MS.}

Sample preparation and quantification of TMA, TMAO, gBB, carnitine, $d_{9^{-}} T M A, d_{g^{-}} T M A O, d_{9}-g B B, d_{9^{-}}$ carnitine in mice

For sample preparation, $50 \mu \mathrm{L}$ of mouse serum was added to $450 \mu \mathrm{L}$ of methanol containing isotopically labeled internal standards $\left(d_{3}\right.$-carnitine, $d_{9}-T M A O$, and ${ }^{13} C_{3}$-TMAO). Subsequently, the mixture was centrifuged at $12,000 \times g, 4^{\circ} \mathrm{C}$ for $5 \mathrm{~min}$, and the supernatants were collected for LC-MS.

For LC-MS analysis, the target metabolites of the serum samples were analyzed using the Agilent 1290 UHPLC tandem Agilent 6460 triple quadrupole mass spectrometer. The MicroSolv Cogent Diamond Hydride column $(150 \times 2.1 \mathrm{~mm}, 4.2 \mu \mathrm{m}$, MicroSolv, Eatontown, $\mathrm{NJ})$ was used in this study by maintaining the column temperature at $40{ }^{\circ} \mathrm{C}$. Mobile phase solution A was $10 \mathrm{mM}$ ammonium acetate, $0.2 \%$ formic acid in deionized water; solution B was $10 \mathrm{mM}$ ammonium acetate and $0.2 \%$ formic acid in $90 \%$ acetonitrile. The flow rate was $0.4 \mathrm{~mL} / \mathrm{min}$, and the gradient program was as follows: $90-75 \%$ solution $B$ for $0-1 \mathrm{~min} ; 75-65 \%$ solution $B$ for $1-2 \mathrm{~min} ; 65-55 \%$ solution $B$ for $2-4 \mathrm{~min} ; 55-40 \%$ solution B in $4-$ 5 min, followed by re-equilibration of the column with $90 \%$ solution $B$ for 1 min.

For MS, the positive electrospray ionization mode was used with the following parameters: drying gas temperature was set at $325^{\circ} \mathrm{C}$, flow rate $7 \mathrm{~L} / \mathrm{min}$, nebulizer pressure at $45 \mathrm{psi}$, sheath gas temperature at $325^{\circ} \mathrm{C}, 11 \mathrm{~L} /$ min sheath gas of flow rate, the capillary voltage at $3,500 \mathrm{~V}$, and nozzle voltage at $500 \mathrm{~V}$. The mass spectrometer was configured in multiple reaction monitoring mode. The monitored transitions for carnitine were: $\mathrm{m} / \mathrm{z} 162.1 \rightarrow 43.2$ and $162.1 \rightarrow 60.2 ; \mathrm{d}_{3}$-carnitine, $\mathrm{m} / \mathrm{z} 165.1 \rightarrow 43.1$ and $165.1 \rightarrow$ 61.2; TMAO, $\mathrm{m} / \mathrm{z} 76.1 \rightarrow 58.1$ and $76.1 \rightarrow 59.1 ; \mathrm{d}_{9}$-TMAO, $\mathrm{m} / \mathrm{z} 85.1 \rightarrow 66.3$ and $85.1 \rightarrow 68.3 ;{ }^{13} \mathrm{C}_{3}$-TMAO, $\mathrm{m} / \mathrm{z} 79.1 \rightarrow 61.1$ and $79.1 \rightarrow 62.1 ; \mathrm{TMA}, \mathrm{m} / \mathrm{z}$ 60.1 $\rightarrow 45.2$ and $60.1 \rightarrow 44.1 ; \mathrm{d}_{9}-\mathrm{TMA}, \mathrm{m} / \mathrm{z} 69.1 \rightarrow 51.1$ and $69.1 \rightarrow 49.1$; and ${ }^{13} \mathrm{C}_{3}$-TMA, $\mathrm{m} / \mathrm{z} 63.1 \rightarrow 46.1$. The detected peak area ratio was used to calculate the concentration of each target analyte in the serum sample against the calibration curve. 
Sample preparation and quantification for TMA, TMAO, gBB, carnitine, choline, $d_{9}-T M A, d_{9}-T M A O, d_{9}-g B B$, $d_{9}$-carnitine in human and culture medium

For sample preparation. $1 \mu \mathrm{L}$ of plasma, urine, or culture medium was mixed with $199 \mu \mathrm{L}$ of isotopically labeled internal standards $\left(d_{3}\right.$-carnitine, $d_{9}$-TMAO, and ${ }^{13} \mathrm{C}_{3}$-TMAO) in $0.1 \%$ formic acid acetonitrile solution. The solution was subsequently centrifuged at $15,000 \mathrm{rpm}, 0{ }^{\circ} \mathrm{C}$ for $5 \mathrm{~min}$. For urine sample preparation, the urine was first centrifuged at $3,000 \times \mathrm{g}, 22^{\circ} \mathrm{C}$ for $15 \mathrm{~min}$. We mixed $5 \mu \mathrm{L}$ of the $1 \mathrm{st}$ supernatant obtained with $45 \mu \mathrm{L}$ of isotopically labeled internal standards $\left(d_{3}\right.$-carnitine, $d_{9}$-TMAO, and ${ }^{13} \mathrm{C}_{3}$-TMAO) in $0.1 \%$ formic acid acetonitrile solution. The solution was centrifuged at $15,000 \mathrm{rpm}, 0{ }^{\circ} \mathrm{C}$ for $5 \mathrm{~min}$, and the $2 \mathrm{nd}$ supernatant was obtained. Next, $2 \mu \mathrm{L}$ of the $2 \mathrm{nd}$ supernatant was mixed with 198 $\mu \mathrm{L}$ of internal standard and centrifuged at $15,000 \mathrm{rpm}, 0{ }^{\circ} \mathrm{C}$ for $5 \mathrm{~min}$; the supernatant obtained was ready for LC-MS/MS quantification. The prepared plasma and urine samples were analyzed using LC-MS/MS SCIEX 5500 for TMA, TMAO, gBB, carnitine, and choline quantification.

For LC-MS/MS analysis, $20 \mu \mathrm{L}$ of each plasma or urine sample was injected into a Sciex Exion LC AC system coupled with a SCIEX Triple TOF 5600 mass spectrometer (AB SCIEX, Canada). The separation was performed using an HILIC column $\left(250 \times 4.0 \mathrm{~mm}, 5 \mu \mathrm{m}\right.$, Fortis, UK) maintained at $40{ }^{\circ} \mathrm{C}$. Mobile phase $A$ was $0.1 \%$ formic acid in deionized water, and mobile phase $B$ was $0.1 \%$ formic acid in acetonitrile. The flow rate was $0.5 \mathrm{~mL} / \mathrm{min}$. The LC program: $0-1 \mathrm{~min}, 50 \%$ solvent $\mathrm{B}, 1-9 \mathrm{~min}, 50-40 \%$ solvent $B, 9-10 \mathrm{~min}, 40 \%$ solvent $B, 10-10.1 \mathrm{~min}$, and $40-50 \%$ solvent $B$, followed by column reequilibration with $50 \%$ solvent $B$ for $1.9 \mathrm{~min}$. The electrospray was set in positive ionization mode with the following parameters: curtain gas supply, $30 \mathrm{psi}$; capillary temperature, $500{ }^{\circ} \mathrm{C}$; spray voltage floating, $5,500 \mathrm{~V}$; and declustering potential, $80 \mathrm{~V}$. The concentration of each analyte was calculated from calibration curves relating the peak area ratio to its corresponding standard.

\section{Platelet Preparation and Aggregometry Assays}

Platelet preparation and aggregometry assays were performed according to the methods used by Zhu et al. (2016) with modifications. ${ }^{16}$ Whole blood was collected from study participants using $3.2 \%$ sodium citrate anticoagulant. Platelet-rich plasma (PRP) was obtained by centrifugation at $100 \times g, 22^{\circ} \mathrm{C}$ for 6 min. Platelet poor plasma (PPP) was prepared by further centrifugation at 11,000 $\times g$ for 2 min. Platelets were counted using Sysmex K1000 Hematology Analyzer, and the CHRONO-LOG490-4D system was used for aggregometry assays. Platelet concentrations were adjusted to $2 \times 108 / \mathrm{mL}$ with PPP. $5 \mu \mathrm{M}$ ADP was used as indicated to initiate aggregation with constant stirring $(600 \mathrm{rpm})$. Both PRP and PPP samples were kept at $37^{\circ} \mathrm{C}$ before the aggregometry assays were performed.

\section{Fecal DNA Extraction and 16S rRNA amplicon sequencing and sequencing analysis}

Fecal sample collection and genomic DNA extraction 
Mouse fecal samples were collected from the large intestine during a sacrifice from both the long-term

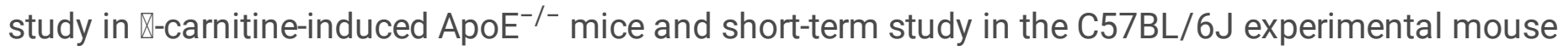
model; the samples were snap frozen using liquid nitrogen and stored at $-80^{\circ} \mathrm{C}$ before use. Human fecal samples were collected before and after the garlic juice intervention using a stool collection kit. ${ }^{55}$ The samples were stored at $-80^{\circ} \mathrm{C}$ for further examination. Fecal genomic DNA was extracted using the QIAmp Power Fecal DNA Kit (QIAGEN, Netherlands) according to the manufacturer's instructions and quantified using the NanoDrop ND-1000 spectrophotometer (Thermo Fischer Scientific).

\section{Polymerase Chain Reaction (PCR), Library Preparation, and 16S rRNA Gene Sequencing}

The V3-V4 hypervariable region of the 16S rRNA gene was amplified using the primer pair ((Forward $=5^{\prime}$ TCG TCG GCA GCG TCA GAT GTG TAT AAG AGA CAG CCT ACG GGN GGC WGC AG-3') and Reverse = 5'GTC TCG TGG GCT CGG AGA TGT GTA TAA GAG ACA GGA CTA CHV GGG TAT CTA ATC C-3')). PCR amplification was performed in a $25 \mu \mathrm{L}$ reaction mixture containing $5 \mathrm{ng}$ of DNA template, $0.2 \mu \mathrm{M}$ forward and reverse primers, and $12.5 \mu \mathrm{L}$ of $2 \times$ Taq Master Mix (KAPA HiFi HotStart ReadyMix, Roche, Switzerland). The PCR conditions involved an initial step at $95^{\circ} \mathrm{C}$ for $3 \mathrm{~min}$, followed by 25 cycles of 95 ${ }^{\circ} \mathrm{C}$ for $30 \mathrm{~s}, 55^{\circ} \mathrm{C}$ for $30 \mathrm{~s}, 72{ }^{\circ} \mathrm{C}$ for $30 \mathrm{~s}$, and a final extension of $72{ }^{\circ} \mathrm{C}$ for $5 \mathrm{~min}$. Subsequently, $2 \%$ agarose gel electrophoresis was used to visualize the amplified products. Dual index and Illumina sequencing adapters were attached by using a Nextera XT Index Kit via PCR according to the manufacturer's instructions. PCR product cleanup was conducted using AMPure XP beads to purify the V3-V4 amplicon. The sizes of PCR products were confirmed using the Bioanalyzer DNA 1000 chip. Library quantification was conducted for quality control before sequencing using the Agilent Technologies 2100 Bioanalyzer. The pooled libraries were subjected to paired-end sequencing $(2 \times 300 \mathrm{bps})$ using the Illumina MiSeq platform.

\section{Sequencing Analysis}

The raw sequences were processed following the instructions in the 16S Bacteria/Archaea SOP v1 of the Microbiome Helper workflows (https://github.com/mlangill/microbiome_helper). ${ }^{56}$ The paired-end reads were filtered as a sequence length of over 400 and a quality score of $90 \%$ at a Phed score 30 , followed by the removal of the chimeric sequences using VSEARCH v2.1.2. ${ }^{57}$ The high-quality reads were subsequently analyzed using QIIME v.1.9.1. ${ }^{58}$ OTUs were produced using the UCLUST algorithm and a closed-referenced OTU approach against the SILVA database (version 132) with $97 \%$ of sequence identity, followed by rarefaction. The vegan package in $\mathrm{R}$ was used to calculate a-diversity indices, including the observed OTUs, Shannon index, and Chao1 index, PCoA based on the Bray-Curtis distance. We performed permutation multivariate analysis of variance (ANOVA) using distance matrices (adonis) to determine the heterogeneity of the fecal microbiota among the groups. A heatmap was plotted using the heatmap3 package.

\section{Statistical Analysis}

Page $15 / 26$ 
Data are represented as the mean \pm standard error of the mean (SEM) or mean \pm standard deviation. An unpaired two-tailed Student's t-test or one-way ANOVA with Tukey's range test was used to compare group means in an animal study. For the human study, data are expressed as the mean \pm SEM; paired two-tailed Student's t-test was used to compare the difference between before and after garlic juice intake. The Kruskal-Wallis test, Wilcoxon signed-rank test with or without false discovery rate, unpaired/paired two-tailed Student's t-test, and one-way ANOVA with Tukey's range test were used for the analysis of the fecal microbiome data based on the data set. All statistics were analyzed using Graphpad Prism (version 8.4.3), $\mathrm{R}$ (version 3.6.1), or R Studio (version 1.2.5001).

\section{Declarations}

\section{Data availability}

The raw 16s rRNA sequencing data used to produce all figures are accessible at the NCBI Short Read Archive under the following accession numbers: BioProject: PRJNA661156 and SRA: SRS7316558, SRS7317051, and SRS7315455.

\section{Acknowledgements}

This study was financed by the Ministry of Science and Technology (Taiwan) (107-2321-B-002-017, 1082321-B-002-035, 107-2321-B-002-039, and 108-2321-B-002-051).

\section{Author contributions}

S.P. instructed and assisted the experiment, performed the bioinformatics and statistical analysis, and drafted the manuscript; W.K.W. designed and instructed the animal and human experiments; K.V.C. performed the human and in vitro studies; P.C.C. performed the animal experiments; Y.T.Y. conducted the PCR and library preparation for 16 S rRNA gene sequencing; H.L.C. guided the animal studies; C.C.C. assisted the human study; R.A.C. and H.S.H. assisted the human study and animal experiments; P.Y.L. assisted the bioinformatics analysis; C.H.C. assisted platelet preparation and aggregometry assays; A.Y.C.L. and C.C.H. instructed target metabolomics analysis and assisted with mass spectrometry analysis; K.C.Y. and T.F.H provided the technical support in the $\mathrm{ApoE}^{-/-}$experiment and aortic lesion investigation; T.C.D.S., H.L.K., C.T.H. and A.N.O. critically reviewed the manuscript; M.S.W. and L.Y.S. designed the experiments, provided the funding for the study, and critically revised the manuscript.

\section{Competing interests}

The authors declare that there are no competing interests.

\section{References}

1. Roth, G. A. et al. Global, regional, and national burden of cardiovascular diseases for 10 causes, 1990 to 2015. J. Am. Coll. Cardiol. 70, 1-25 (2017). 
2. Zhao, D., Liu, J., Wang, M., Zhang, X. \& Zhou, M. Epidemiology of cardiovascular disease in china: Current features and implications. Nat. Rev. Cardiol. 16, 203-212 (2019).

3. Noble, N., Paul, C., Turon, H. \& Oldmeadow, C. Which modifiable health risk behaviours are related? A systematic review of the clustering of smoking, nutrition, alcohol and physical activity ('snap') health risk factors. Prev. Med. 81, 16-41 (2015).

4. Clarke, G., Stilling, R. M., Kennedy, P. J., Stanton, C., Cryan, J. F. \& Dinan, T. G. Minireview: Gut microbiota: The neglected endocrine organ. Mol. Endocrinol. 28, 1221-1238 (2014).

5. David, L. A. et al. Diet rapidly and reproducibly alters the human gut microbiome. Nature 505, 559563 (2014).

6. Jie, Z. et al. The gut microbiome in atherosclerotic cardiovascular disease. Nat. Commun. 8, 845 (2017).

7. Brown, J. M. \& Hazen, S. L. The gut microbial endocrine organ: Bacterially derived signals driving cardiometabolic diseases. Annu. Rev. Med. 66, 343-359 (2015).

8. Gentile, C. L. \& Weir, T. L. The gut microbiota at the intersection of diet and human health. Science $362,776-780$ (2018).

9. Witkowski, M., Weeks, T. L. \& Hazen, S. L. Gut microbiota and cardiovascular disease. Circ. Res. $127,553-570$ (2020).

10. Koeth, R. A. et al. Intestinal microbiota metabolism of I-carnitine, a nutrient in red meat, promotes atherosclerosis. Nat. Med. 19, 576-585 (2013).

11. Tilg, H. A gut feeling about thrombosis. New Engl. J. Med. 374, 2494-2496 (2016).

12. Wang, Z. et al. Gut flora metabolism of phosphatidylcholine promotes cardiovascular disease. Nature 472, 57-63 (2011).

13. Schiattarella, G. G. et al. Gut microbe-generated metabolite trimethylamine-N-oxide as cardiovascular risk biomarker: A systematic review and dose-response meta-analysis. Eur. Heart. J. 38, 2948-2956 (2017).

14. Tang, W. H. et al. Intestinal microbial metabolism of phosphatidylcholine and cardiovascular risk. New Eng. J. Med. 368, 1575-1584 (2013).

15. Zhu, Y. et al. Carnitine metabolism to trimethylamine by an unusual rieske-type oxygenase from human microbiota. Proc. Natl. Acad. Sci. U. S. A. 111, 4268-4273 (2014).

16. Zhu, W. et al. Gut microbial metabolite tmao enhances platelet hyperreactivity and thrombosis risk. Cell 165, 111-124 (2016). 
17. Zhu, W. F., Wang, Z. N., Tang, W. H. W. \& Hazen, S. L. Gut microbe-generated trimethylamine n-oxide from dietary choline is prothrombotic in subjects. Circulation 135, 1671-1673 (2017).

18. Koeth, R. A. et al. L-carnitine in omnivorous diets induces an atherogenic gut microbial pathway in humans. J. Clin. Invest. 129, 373-387 (2019).

19. Koeth, R. A. et al. $\gamma$-butyrobetaine is a proatherogenic intermediate in gut microbial metabolism of L-carnitine to TMAO. Cell Metab. 20, 799-812 (2014).

20. Wu, W. K. et al. Characterization of TMAO productivity from carnitine challenge facilitates personalized nutrition and microbiome signatures discovery. Microbiome 8, 162 (2020).

21. Barabási, A.-L., Menichetti, G. \& Loscalzo, J. The unmapped chemical complexity of our diet. Nat. Food 1, 33-37 (2020).

22. Roberts, A. B. et al. Development of a gut microbe-targeted nonlethal therapeutic to inhibit thrombosis potential. Nat. Med. 24, 1407-1417 (2018).

23. Smits, L. P. et al. Effect of vegan fecal microbiota transplantation on carnitine- and choline-derived trimethylamine-N-oxide production and vascular inflammation in patients with metabolic syndrome. $J$. Am. Heart Assoc. 7, (2018).

24. Wang, Z. et al. Non-lethal inhibition of gut microbial trimethylamine production for the treatment of atherosclerosis. Cell 163, 1585-1595 (2015).

25. Petrovska, B. B. \& Cekovska, S. Extracts from the history and medical properties of garlic. Pharmacogn. Rev. 4, 106-110 (2010).

26. Banerjee, S. K. \& Maulik, S. K. Effect of garlic on cardiovascular disorders: A review. Nutr. J. 1, 4 (2002).

27. Liperoti, R., Vetrano, D. L., Bernabei, R. \& Onder, G. Herbal medications in cardiovascular medicine. J. Am. Coll. Cardiol. 69, 1188-1199 (2017).

28. Rahman, K. \& Lowe, G. M. Garlic and cardiovascular disease: A critical review. J. Nutr. 136, 736740 (2006).

29. Sobenin, I. A., Pryanishnikov, V. V., Kunnova, L. M., Rabinovich, Y. A., Martirosyan, D. M. \& Orekhov, A. N. The effects of time-released garlic powder tablets on multifunctional cardiovascular risk in patients with coronary artery disease. Lipids Health Dis. 9, (2010).

30. Harris, J. C., Cottrell, S. L., Plummer, S. \& Lloyd, D. Antimicrobial properties of Allium sativum (garlic). Appl. Microbiol. Biotechnol. 57, 282-286 (2001). 
31. Ankri, S. \& Mirelman, D. Antimicrobial properties of allicin from garlic. Microbes Infect. 1, 125-129 (1999).

32. Choo, S. et al. Review: Antimicrobial properties of allicin used alone or in combination with other medications. Folia. Microbiol. 65, 451-465 (2020).

33. Reiter, J., Levina, N., van der Linden, M., Gruhlke, M., Martin, C. \& Slusarenko, A. J. Diallylthiosulfinate (allicin), a volatile antimicrobial from garlic (Allium sativum), kills human lung pathogenic bacteria, including MDR strains, as a vapor. Molecules 22, (2017).

34. Panyod, S. \& Sheen, L. Y. Beneficial effects of chinese herbs in the treatment of fatty liver diseases. J. Tradit. Complement. Med. 10, 260-267 (2020).

35. Panyod, S. et al. Allicin modifies the composition and function of the gut microbiota in alcoholic hepatic steatosis mice. J. Agric. Food. Chem. 68, 3088-3098 (2020).

36. Wu, W. K., Panyod, S., Ho, C. T., Kuo, C. H., Wu, M. S. \& Sheen, L. Y. Dietary allicin reduces transformation of L-carnitine to TMAO through impact on gut microbiota. J. Funct. Foods 15, 408-417 (2015).

37. Wu, W.-K. et al. Identification of TMAO-producer phenotype and host-diet-gut dysbiosis by carnitine challenge test in human and germ-free mice. Gut 68, 1439-1449 (2019).

38. Kasahara, K. et al. Interactions between Roseburia intestinalis and diet modulate atherogenesis in a murine model. Nat Microbio/ 3, 1461-1471 (2018).

39. Li, J., Lin, S., Vanhoutte, P. M., Woo, C. W. \& Xu, A. Akkermansia muciniphila protects against atherosclerosis by preventing metabolic endotoxemia-induced inflammation in $\mathrm{ApoE}^{-/-}$mice. Circulation 133, 2434-2446 (2016).

40. Chen, K. et al. Preventive effects and mechanisms of garlic on dyslipidemia and gut microbiome dysbiosis. Nutrients 11, (2019).

41. O'Gara, E. A., Hill, D. J. \& Maslin, D. J. Activities of garlic oil, garlic powder, and their diallyl constituents against Helicobacter pylori. Appl. Environ. Microb. 66, 2269-2273 (2000).

42. Naganawa, R., Iwata, N., Ishikawa, K., Fukuda, H., Fujino, T. \& Suzuki, A. Inhibition of microbial growth by ajoene, a sulfur-containing compound derived from garlic. Appl. Environ. Microb. 62, 42384242 (1996).

43. Casella, S., Leonardi, M., Melai, B., Fratini, F. \& Pistelli, L. The role of diallyl sulfides and dipropyl sulfides in the in vitro antimicrobial activity of the essential oil of garlic, Allium sativum L., and leek, Allium porrum L. Phytother. Res. 27, 380-383 (2013). 
44. Avato, P., Tursi, F., Vitali, C., Miccolis, V. \& Candido, V. Allylsulfide constituents of garlic volatile oil as antimicrobial agents. Phytomedicine 7, 239-243 (2000).

45. Everard, A. et al. Cross-talk between Akkermansia muciniphila and intestinal epithelium controls diet-induced obesity. Proc. Natl Acad. Sci. U. S. A. 110, 9066-9071 (2013).

46. Chelakkot, C. et al. Akkermansia muciniphila-derived extracellular vesicles influence gut permeability through the regulation of tight junctions. Exp. Mol. Med. 50, (2018).

47. Sokol, H. et al. Faecalibacterium prausnitzii is an anti-inflammatory commensal bacterium identified by gut microbiota analysis of crohn disease patients. Proc. Natl Acad. Sci. U. S. A. 105, 1673116736 (2008).

48. Panyod, S. et al. Diet supplementation with allicin protects against alcoholic fatty liver disease in mice by improving anti-inflammation and antioxidative functions. J. Agric. Food Chem. 64, 7104-7113 (2016).

49. Beattie, J. H., Duthie, S. J., Kwun, I. S., Ha, T. Y. \& Gordon, M. J. Rapid quantification of aortic lesions in $\mathrm{ApoE}^{(--)}$mice. J. Vasc. Res. 46, 347-352 (2009).

50. Lai, Y. S. et al. Garlic essential oil protects against obesity-triggered nonalcoholic fatty liver disease through modulation of lipid metabolism and oxidative stress. J. Agric. Food Chem. 62, 5897-5906 (2014).

51. Goodman, A. L. et al. Extensive personal human gut microbiota culture collections characterized and manipulated in gnotobiotic mice. Proc. Natl Acad. Sci. U. S. A. 108, 6252-6257 (2011).

52. Krautkramer, K. A. et al. Diet-microbiota interactions mediate global epigenetic programming in multiple host tissues. Mol. Cell. 64, 982-992 (2016).

53. Mortele, O. et al. Optimization of an in vitro gut microbiome biotransformation platform with chlorogenic acid as model compound: From fecal sample to biotransformation product identification. J. Pharmaceut. Biomed. 175, (2019).

54. Romano, K. A., Vivas, E. I., Amador-Noguez, D. \& Rey, F. E. Intestinal microbiota composition modulates choline bioavailability from diet and accumulation of the proatherogenic metabolite trimethylamine-N-oxide. mBio 6, e02481 (2015).

55. Wu, W. K. et al. Optimization of fecal sample processing for microbiome study - the journey from bathroom to bench. J. Formos. Med. Assoc. 118, 545-555 (2019).

56. Comeau, A. M., Douglas, G. M. \& Langille, M. G. Microbiome helper: A custom and streamlined workflow for microbiome research. mSystems 2, (2017). 
57. Rognes, T., Flouri, T., Nichols, B., Quince, C. \& Mahe, F. Vsearch: A versatile open source tool for metagenomics. PeerJ 4, e2584 (2016).

58. Caporaso, J. G. et al. Qiime allows analysis of high-throughput community sequencing data. Nat. Methods 7, 335-336 (2010).

\section{Figures}

a

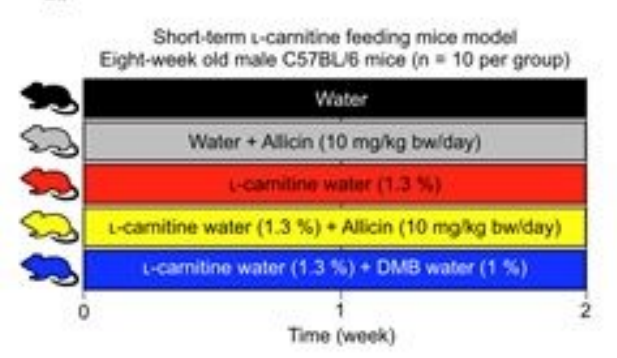

b

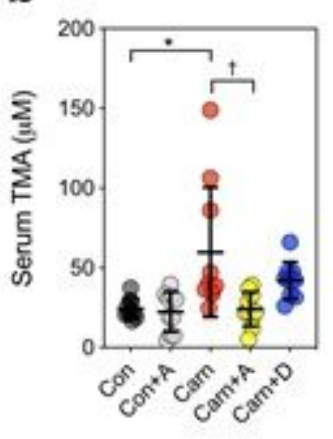

C

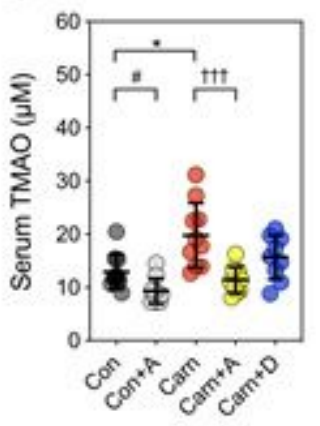

d

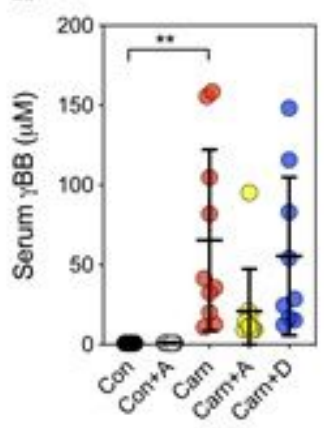

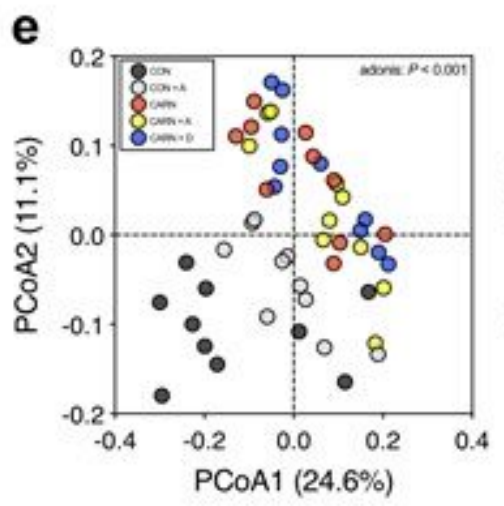

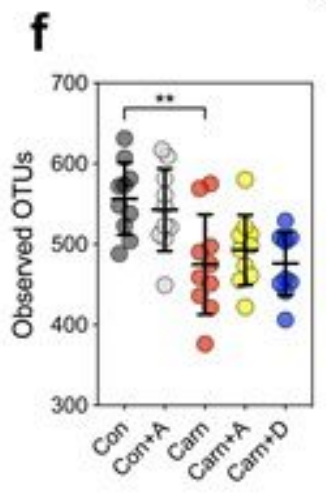

g
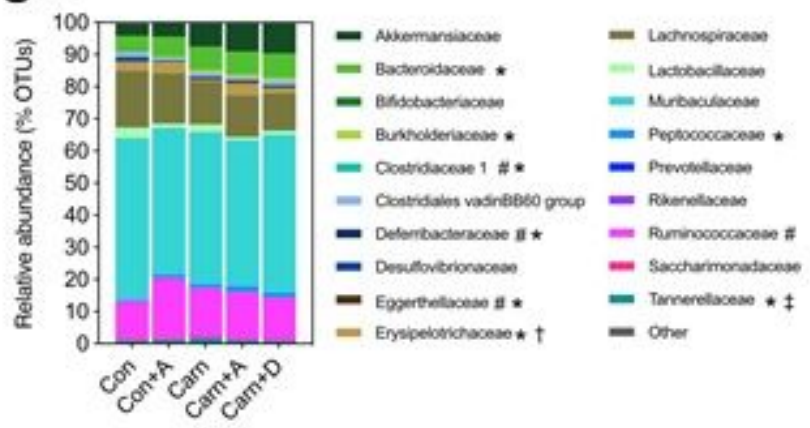

Figure 1

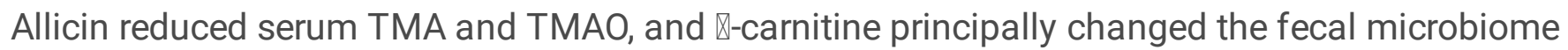

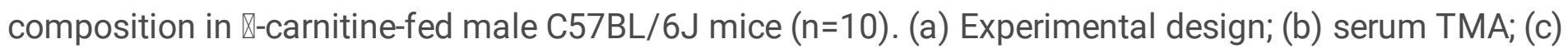
serum TMAO; (d) serum YBB; (e) principal coordinate analysis ( $P C O A$ ) plot with Bray-Curtis dissimilarity; (f) observed OTUs a-diversity indices; and (g) relative abundance of fecal microbiota at family level. Scatter plots are expressed as the mean $\pm S D$; Statistical analyses were performed using an unpaired twotailed Student's t-test control vs control + allicin group $(\#, p<0.05)$, control vs carnitine group $\left({ }^{*}, p<0.05\right.$; and $* *, p<0.01)$; one-way ANOVA with Tukey's range test for comparisons, carnitine vs carnitine + allicin group $(t, p<0.05$; and $+\dagger+, p<0.001)$. The relative abundance bar plot statistical analyses were performed using an unpaired Wilcoxon signed-rank test with the false discovery rate (FDR), control vs control + allicin group (\#, $\mathrm{q}<0.1)$; control vs carnitine group $\left({ }^{*}, \mathrm{q}<0.1 ;\right)$; carnitine vs carnitine + allicin group $(\dagger, q<0.1)$; carnitine + DMB group $(\ddagger, q<0.1)$. 
a

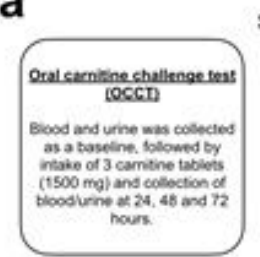

b

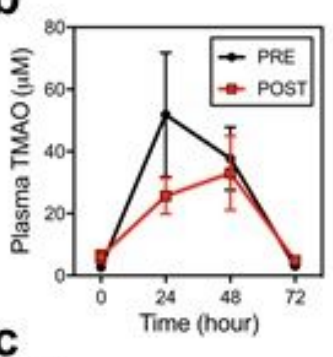

C
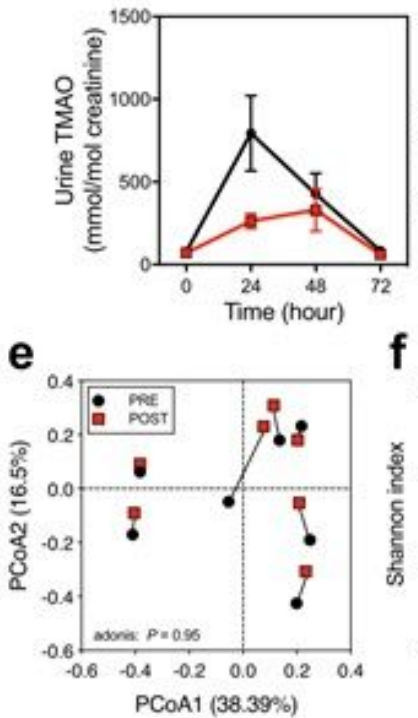

f

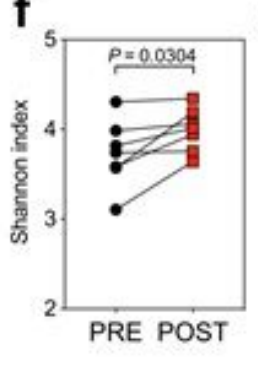

g

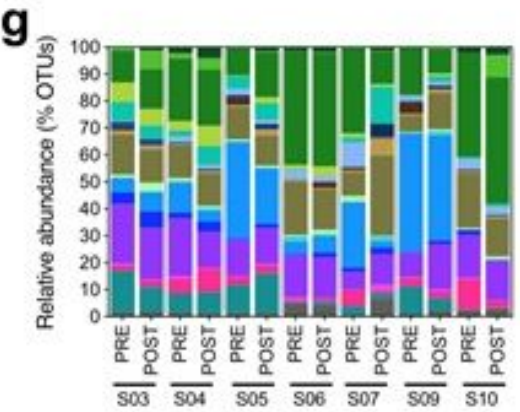

h

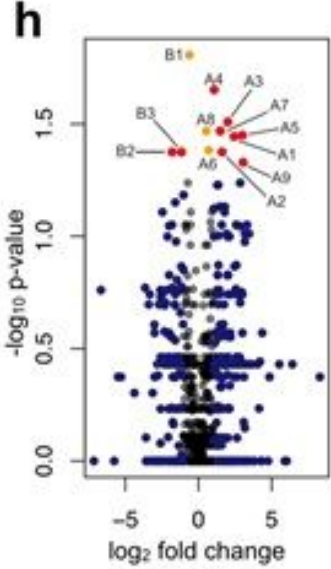

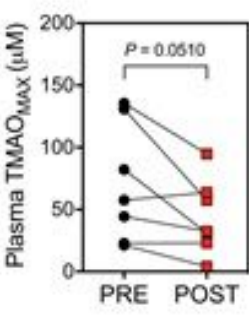
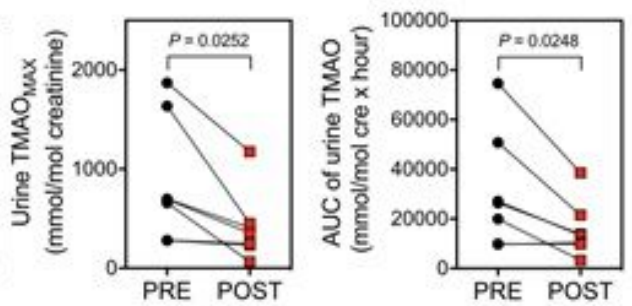

$\frac{10}{S 00} \frac{104}{S 05} \frac{a}{S 06} \frac{207}{S 09} \frac{a}{S 10}$

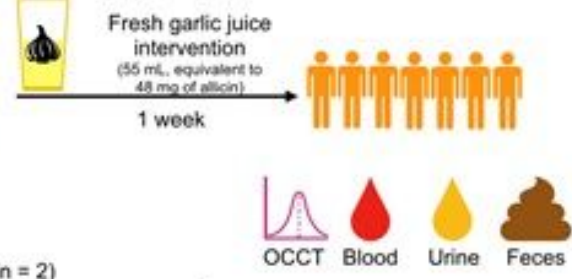

d
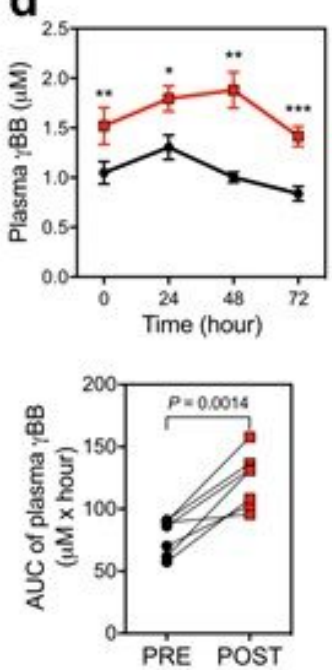

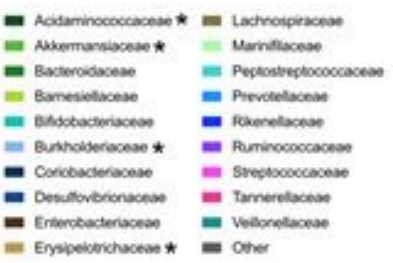

= Enspelionichacesos * = Ore

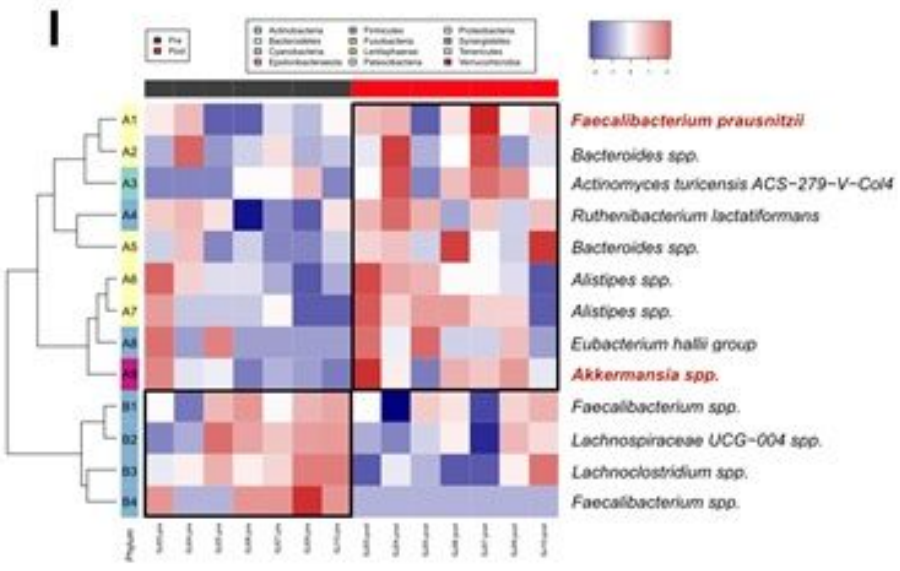

Figure 2

Raw garlic juice reduced plasma TMAO formation ability, increased plasma YBB level in healthy TMAOproducers, and shaped fecal microbiota composition via increasing the evenness, alpha diversity and relative abundance of specific beneficial bacterial taxa. (a) Experimental design, healthy participants $(\mathrm{n}=$ 9) were screened for TMAO production using oral carnitine challenge test (OCCT), the criterion for categorization as high-TMAO producers was plasma TMAOMAX > 10 $\mu$ M. High-TMAO producers $(n=7)$ 
subsequently received an intervention of garlic juice $(55 \mathrm{~mL}$, equivalent to $48 \mathrm{mg}$ of allicin/day) for 1 week, followed by OCCT; (b) plasma and (c) urine TMAO, TMAOMAX, and TMAOAUC; (d) plasma $y B B$ and YBBAUC. (e) Principal coordinate analysis (PCoA) plot with Bray-Curtis dissimilarity; (f) Shannon adiversity index; (g) family-level composition of fecal microbiome; $(\mathrm{h})$ volcano plot of fecal microbiota before and after garlic juice intervention; and (i) heat map of the relative abundances of fecal microbiota with a significant difference using the Wilcoxon signed-rank test $(q<0.05)$. Data are expressed as the mean $\pm S E M$; statistical analysis were performed by using two-tailed paired Student's t-test. The relative abundance bar plot statistical analysis was performed using a paired Wilcoxon signed-rank test $\left({ }^{*}, \mathrm{p}<\right.$ 0.05). 

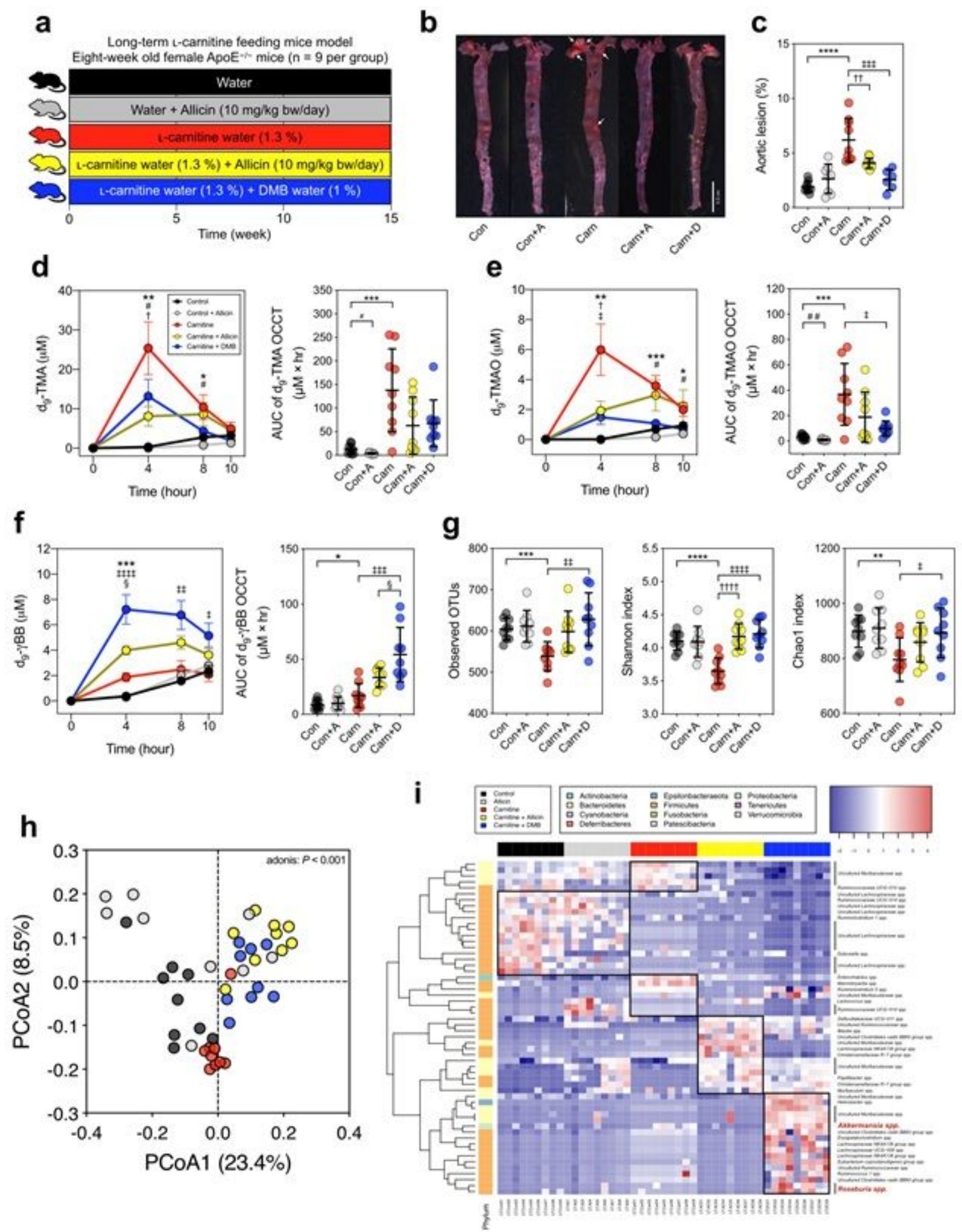

\section{Figure 3}

Allicin reduced aortic lesions through the reduction of TMA and TMAO formation and changed the fecal

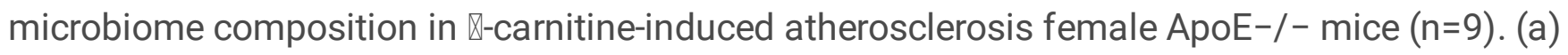
experimental design; (b) representative image of oil red-stained aorta; (c) percentage of aortic lesion; (d) d9-TMA level according to oral carnitine challenge test (OCCT) and its AUC; (e) d9-TMAO level of OCCT and its AUC; (f) d9-үBB level according to OCCT and its AUC. (g) a-diversity indices, observed OTUs, 
Shannon index and Chao1 index; (h) Principal coordinate analysis (PCoA) plot with Bray-Curtis dissimilarity; and (i) heat map of the relative abundances of fecal microbiota with a significant difference using the Kruskal-Wallis test with false discovery rate (FDR) $(q<0.001)$. OCCT curves are expressed as the mean $\pm S E M$, and scatter plots are expressed as the mean $\pm S D$; Statistical analyses were performed using an unpaired two-tailed Student's t-test control vs control + allicin group $(\#, p<0.05$, and \#\#, $p<$ $0.01)$, control vs carnitine group $(*, p<0.05 ; * \star, p<0.01$; $* \star *, p<0.001$; and $* \star \star \star, p<0.0001)$; one-way ANOVA with Tukey's range test for comparisons, carnitine vs carnitine + allicin group $(\dagger, p<0.05 ; \uparrow+, p<$

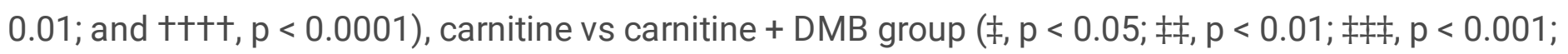

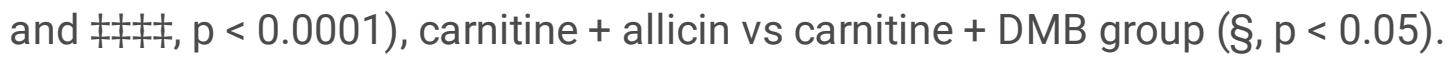

a

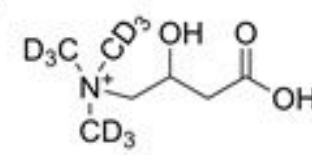

dg-carnitine

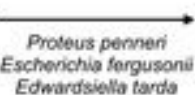

Edwardsiello tards<smiles>O=C(O)CCCC(=O)[N+]([O-])([O-])[O-]</smiles>

dg-YBB
$\mathrm{D}_{3} \mathrm{C}, \mathrm{CD}_{3}$

${ }^{+} \mathrm{NH}$

$\mathrm{CD}_{3}$

do-TMA

\section{b}
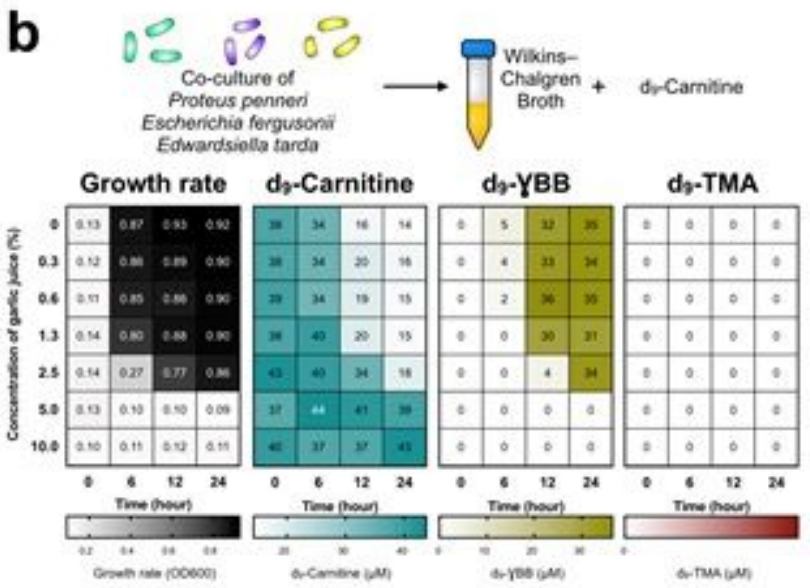

d
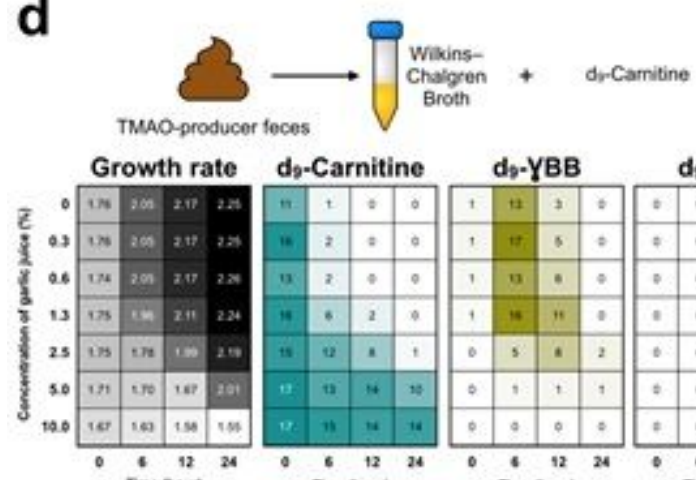

$d_{0}-Y_{B B}$

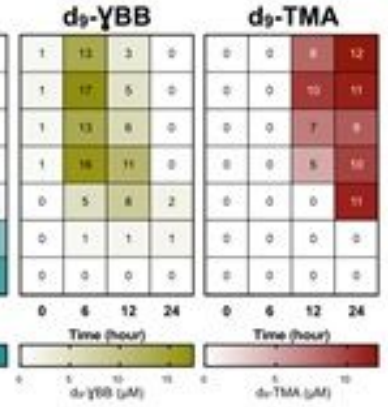

C
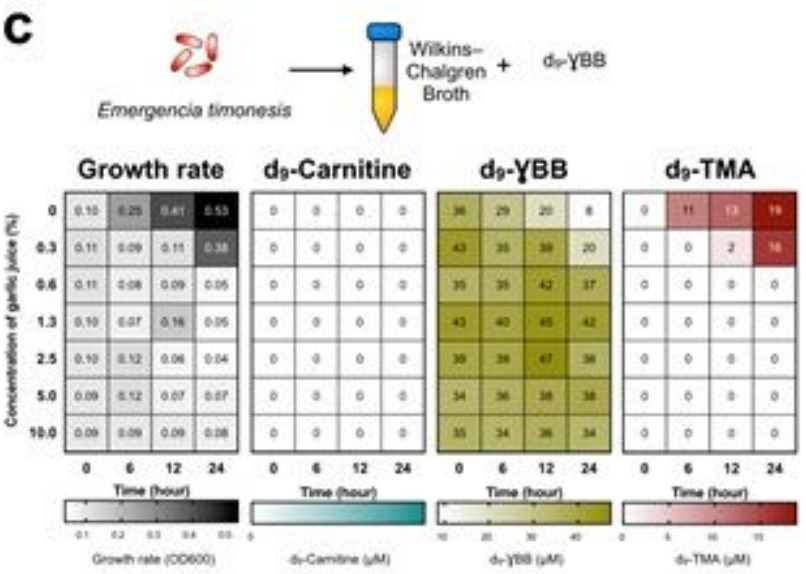

e
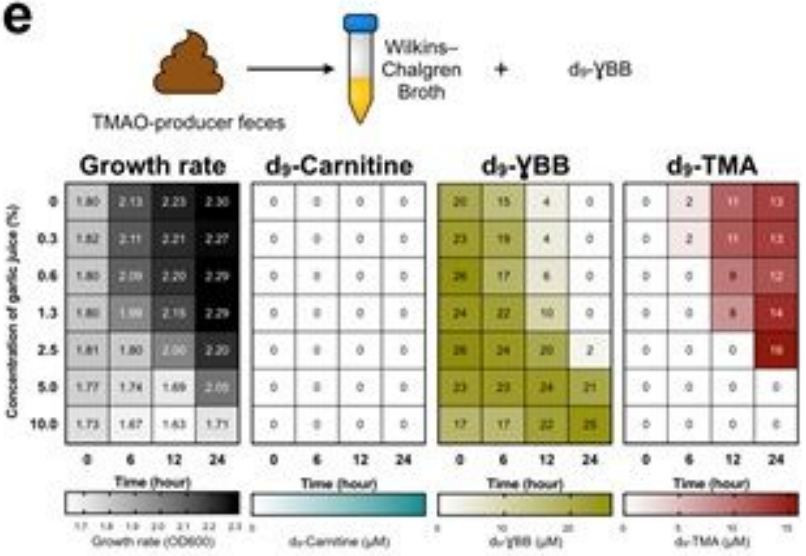

Figure 4

Garlic juice inhibited the formation of d9-TMA and d9-yBB in vitro after inoculation of TMA/үBBproducing bacteria or the feces from high-TMAO producers. (a) Schematic diagram of the bacteria responsible for metabolite d9-carnitine $\rightarrow d 9-\gamma B B \rightarrow d 9-T M A ;(b)$ inhibitory effect of garlic juice on carnitine $\rightarrow$ үBB bacteria (co-culture of Proteus penneri, Escherichia fergusonii, and Edwardsiella tarda) in WC medium supplemented with d9-carnitine; (c) inhibitory effect of garlic juice on the bacteria 
responsible for converting YBB to TMA (Emergencia timonensis) in WC medium supplemented with d9YBB. (d) Inhibitory effect of garlic juice on the high-TMAO-producing gut microbiome in WC medium supplemented with d9-carnitine and (e) d9-yBB.

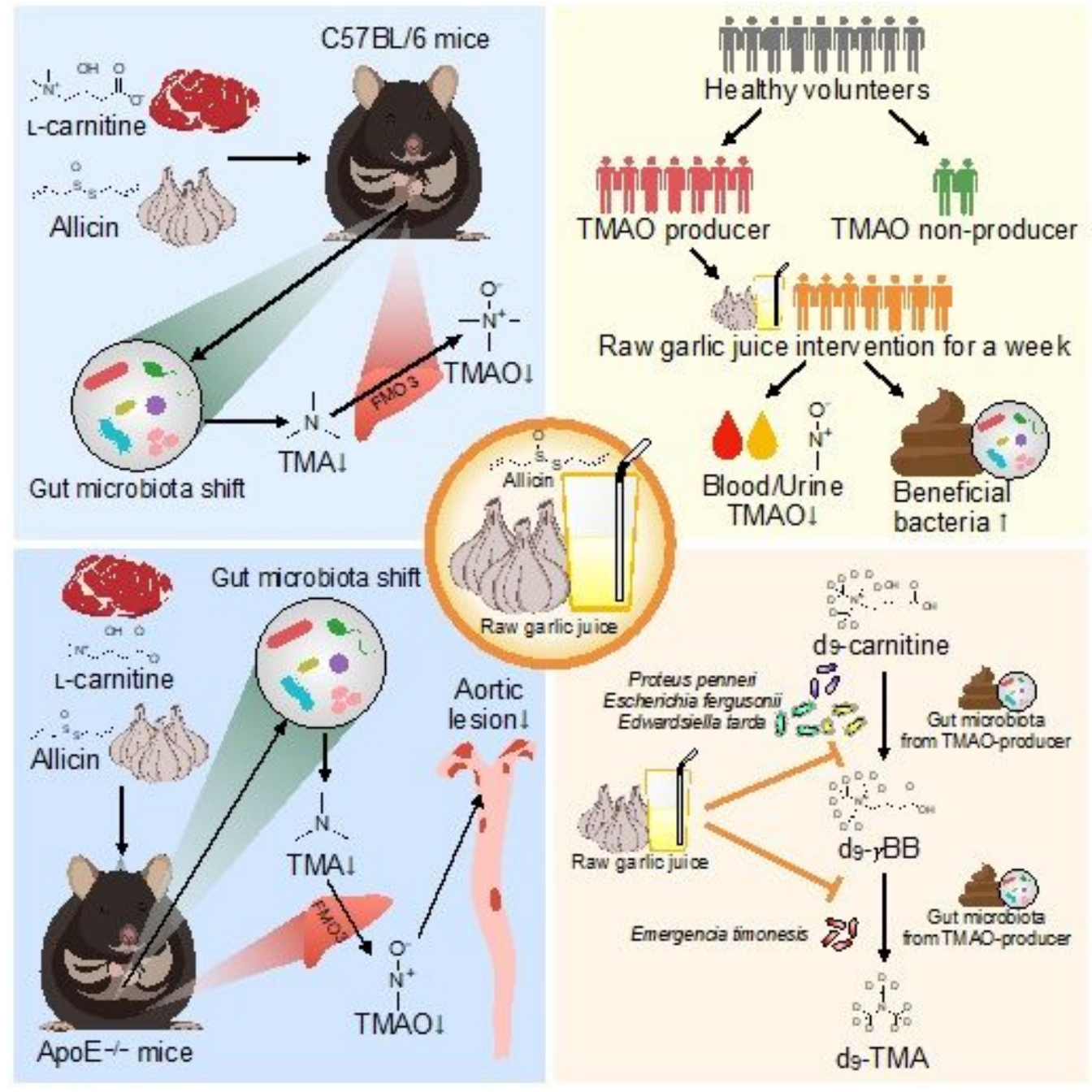

\section{Figure 5}

The role of allicin and raw garlic juice on CVD prevention and atherosclerosis amelioration through gut microbiota and TMAO modulation. (1) Allicin decreased gut microbiota and host-derived TMA and TMAO in mice supplemented with $₫$-carnitine in drinking water; (2) Raw garlic juice reduced plasma and urine TMAO and increased beneficial gut bacteria in high-TMAO producing humans; (3) Allicin ameliorated aortic lesions by reducing TMA/TMAO production and altered the gut microbiome in $\triangle$-carnitine-induced atherosclerosis ApoE-/- mice; (4) Raw garlic juice inhibited microbial pathway of carnitine $\rightarrow$ yBB $\rightarrow$ TMA conversion in specific bacteria and high-TMAO producer's gut microbiome.

\section{Supplementary Files}

This is a list of supplementary files associated with this preprint. Click to download.

- Supplementarynpjbiofilmsandmicrobiomes.docx 